\title{
Impact de la Covid-19 sur les arrêts cardiaques extrahospitaliers en phase de suractivité épidémique : données du registre RéAC
}

\author{
COVID-19 Impact on Out-of-Hospital Cardiac Arrest in Epidemic Overactivity Phase: \\ Data from the RéAC Registry
}

\author{
P.-Y. Gueugniaud · V. Baert · D. Hugenschmitt $\cdot$ H. Hubert $\cdot$ K. Tazarourte \\ Reçu le 15 octobre 2020 ; accepté le 29 octobre 2020 \\ (C) SFMU et Lavoisier SAS 2020
}

Résumé Introduction : Notre objectif était d'analyser la survie des patients victimes d'un arrêt cardiaque extrahospitalier (ACEH) durant la pandémie Covid-19 et de comparer les données en fonction du centre de traitement de l'appel choisi, le 15 ou le 18 .

Méthode : Nous avons extrait les données exhaustives du Registre des arrêts cardiaques (RéAC), entre le $1^{\text {er }}$ mars et le 30 avril 2020. Nous avons effectué trois comparaisons de la survie à 30 jours (J30) de cohortes de patients : 1) Covid vs non-Covid ; 2) appels arrivés au service d'aide médicale urgente (Samu) (15) vs aux sapeurs-pompiers (SP) (18) et 3) appels arrivés au 15 vs 18 pour les patients Covid.

Résultats : Sur un total de 870 ACEH, 184 étaient atteints de la Covid. Nous avons observé 487 (56\%) appels arrivés au 15 et $383(44 \%)$ au 18 . La survie à J30 était de $3 \%$. Les ACEH Covid avaient une survie à $\mathrm{J} 30$ plus faible que les non-Covid ( 0 vs $4 \%, p<0,001)$. Le délai d'arrivée de SP était plus long lors d'un appel au 15. En revanche, aucune différence de survie n'est observée entre les appels arrivés au 15 ou au 18.

Conclusion : La survie consécutive à un ACEH durant la pandémie est extrêmement faible. Cependant, quel que soit le numéro composé (15 ou 18), la survie n'est pas différente, même si le délai d'arrivée des prompts secours est plus court lors d'un appel au 18.

P.-Y. Gueugniaud $(\bowtie) \cdot$ D. Hugenschmitt $\cdot$ K. Tazarourte

Samu 69, groupement hospitalier Édouard-Herriot,

hospices civils de Lyon, université Claude-Bernard-Lyon-I,

F-69437 Lyon, France

e-mail : pierre-yves.gueugniaud@chu-lyon.fr

V. Baert $\cdot$ H. Hubert

Université de Lille, CHU de Lille, ULR 2694-METRICS :

Évaluation des technologies de santé et des pratiques médicales,

F-59000 Lille, France

K. Tazarourte

Laboratoire ESPER, université Claude-Bernard-Lyon-I,

F-69100 Villeurbanne, France
Mots clés Arrêts cardiaques extrahospitaliers · Registre · Covid-19 · SARS-CoV-2 · Centre de traitement des appels . Centre de réception et de régulation des appels

Abstract Introduction: Our aim was to analyze the outof-hospital cardiac arrest (OHCA) care and patients' survival during COVID-19 pandemic by comparing the emergency phone number called to initiate the alert [EMS(\#15) or firefighters(\#18)].

Procedures: We used data from the French OHCA Registry (RéAC), between March 1, 2020 and April 30, 2020. We performed three comparisons of patient cohorts: 1) COVID vs non-COVID; 2) 15 vs 18 calls and 3) in COVID patients, 15 vs 18 calls.

Results: We included 870 OHCA, among them, 184 were affected by COVID-19. There were $487(56 \%)$ calls to 15 and 383 (44\%) to 18 . Patient survival at $\mathrm{D}+30$ during the study was $3 \%$. COVID+ patients had a lower survival rate at $\mathrm{D}+30$ than non-COVID patients $(0 \%$ vs. $4 \%, P<0.001)$. Firefighters had a long time to arrive on the scene for calls to 15 . No difference in survival was observed between 15 or 18 calls.

Conclusion: The poor survival of patients during the pandemic is multi-causal but does not appear to be related to the emergency phone number called to initiate the alert [EMS (\#15) or firefighters (\#18)] even if the arrival time for prompt assistance is shorter on the call to 18 .

Keywords Out-of-hospital cardiac arrest · COVID-19 . Registry $\cdot$ SARS-CoV-2 $\cdot$ Call processing centre

\section{Introduction}

La pandémie de SARS-CoV-2 a conduit à une réorganisation des systèmes de santé pour limiter autant que possible la propagation du virus, et l'importance de la vague 
épidémique initiale du coronavirus a eu un impact considérable sur le système sanitaire français avec des conséquences sur la prise en charge de certaines pathologies [1]. Les systèmes médicaux d'urgence préhospitaliers, incluant les services de réception des appels d'urgences, ont dû s'adapter en permanence tout en faisant face à des stratégies d'accueil des patients et des conditions de travail, difficiles [2]. Les principales mesures adoptées reposent, pour le grand public, sur l'isolement de la population et la distanciation physique et, pour les personnels soignants, sur le port d'équipements de protection individuelle. Le virus est transmis principalement par contact direct ou par gouttelettes [3] de personnes infectées symptomatiques ou non symptomatiques [4]. En dehors de l'épidémie virale, la réanimation cardiopulmonaire (RCP) n'est pas considérée comme une source fréquente de transmission de maladies infectieuses (estimée à moins de 1/200 000) [5], cependant, un contact étroit avec un sujet potentiellement infecté, imposé par la RCP, pourrait être une source de contamination par le SARS-CoV-2 [6,7]. Les directives de réanimation, en vigueur depuis 2015 , ont donc été adaptées à cette nouvelle situation ; par exemple, pour la RCP de base (RCP-B), le bouche-à-bouche en plus de la compression thoracique n'est plus recommandé pour le témoin [8-10]. Pour la RCP spécialisée (RCP-S), la ventilation par masque ou la ventilation par dispositifs supraglottiques sont considérées comme des alternatives acceptables à l'intubation trachéale [11]. Une mise à jour de ces recommandations a été publiée, notamment par l'International Liaison Committee on Resuscitation (ILCOR) [12]. Les principaux changements recommandent que les témoins ne réalisent que des compressions thoraciques, sauf pour les enfants. Toutes les équipes de secouristes et du service mobile d'urgence et de réanimation (Smur) doivent utiliser un équipement de protection individuelle pendant la réanimation. Selon les recommandations françaises, les médecins du Smur doivent favoriser l'intubation orotrachéale précoce afin de minimiser l'aérosolisation [13]. Ces changements ont eu un impact sur la prise en charge des arrêts cardiaques extrahospitaliers (ACEH) à chaque niveau (RCP-B et RCP-S). La qualité de la prise en charge des urgences vitales, et notamment des ACEH, a été discutée et a même fait l'objet de polémiques [14]. Notamment, il a été rapporté que les délais d'attente et d'intervention ont été allongés pendant l'épidémie, lors d'un appel au centre 15 comparativement à un appel au centre de traitement des pompiers, impliquant potentiellement une perte de chance pour les patients.

Dans ce contexte, le but de notre étude était d'analyser la prise en charge des patients victimes d'un ACEH en termes de délais d'intervention et de survie durant l'acmé de la pandémie (mars-avril 2020) et d'évaluer l'influence du centre d'appel choisi pour déclencher l'alerte entre le centre de traitement des appels (CTA) des sapeurs-pompiers (SP) (18 ou
112 ) et le centre de réception et de régulation des appels (CRRA) des services d'aide médicale urgente (Samu) (15).

\section{Méthode}

En France, le système d'urgence préhospitalier comporte deux niveaux [15]. Un premier niveau est mis en œuvre par les SP des services d'incendie et de secours (SIS) qui réalisent les gestes de RCP-B. Le second niveau est déployé par l'équipe médicale du Smur qui réalise une RCP-S. La coordination de ces interventions est sous la responsabilité des CRRA des Samu. Dans le cadre de cette organisation, tous les Smur participant au Registre des ACEH (RéAC) utilisent, dans le cadre de la prise en charge de ces ACEH, une feuille de recueil des données d'intervention spécifique [16]. Cette observation, adaptée au système d'urgence préhospitalier, respecte les consignes réglementaires et suit le style d'Utstein [17]. Les données sont ensuite reportées sur le site sécurisé de RéAC (https://registreac.org). Durant la période de pandémie, les utilisateurs de RéAC ont eu la possibilité de renseigner la présence et/ou la suspicion d'une infection par la Covid-19. Un suivi à 30 jours après l'ACEH ou à la sortie de réanimation est réalisé pour les patients admis en vie à l'hôpital [18].

Le critère de jugement principal de notre étude était le taux de survie à 30 jours ou à la sortie de réanimation (j30). Les critères de jugement secondaires étaient le taux de reprise d'une activité circulatoire spontanée (RACS), la survie à $\mathrm{j} 0$ et pour les patients en vie à $\mathrm{j} 30$, le taux de devenir neurologique favorable à j30, c'est-à-dire l'absence de séquelle neurologique ou des séquelles modérées (correspondant aux catégories CPC 1 et 2 de la Cerebral Performance Category Scale) [19].

Notre étude de cohorte comparative et multicentrique a été fondée sur les données du registre RéAC. Nous avons collecté les données des patients pour lesquels un appel au secours avait été passé au centre 15 ou 18/112 entre le $1^{\text {er }}$ mars et le 30 avril 2020 sans critère d'exclusion. Les ACEH inclus dans l'étude sont ceux issus des Samu/Smur dont l'exhaustivité de l'inclusion des patients en arrêt cardiaque dans le registre suite à un appel au centre 15 ou au 18/112 a pu être vérifiée et dont la qualité des données recueillies a été contrôlée.

Ainsi, le recueil des patients d'un Smur a été qualifié d'exhaustif, si la méthode de capture/recapture par rapport à la base des appels au Samu a été satisfaisante ou si le Smur a attesté l'inclusion systématique de chacun de ses cas d'ACEH. Pour les Smur satisfaisant ces critères, une comparaison du taux d'incidence calculé à partir des données du RéAC et de celui calculé par les Samu a été réalisée. Pour contrôler la qualité des données saisies, plusieurs procédures de contrôle qualité sont utilisées lors de la saisie des données 
pour détecter en temps réel les erreurs de saisie, les incohérences ou les valeurs aberrantes. Des tests a posteriori sont effectués pour détecter d'autres types d'erreurs qui doivent être vérifiées par les investigateurs des centres participants. De plus, des dossiers patients choisis au hasard sont évalués par un attaché de recherche clinique afin d'identifier d'autres incohérences ou erreurs. Par ailleurs, pour cette étude, il fallait que les centres choisis aient renseigné de façon systématique la présence ou l'absence de la Covid-19 durant la période de l'étude. Ainsi, sur un total de $311 \mathrm{Samu} / \mathrm{Smur}$ participants à RéAC, nous avons sélectionné 23 centres $\mathrm{Samu} / \mathrm{Smur}$ français répondants à ces deux conditions (Argenteuil, Bobigny, Corbeil-Essonnes, Créteil, Douai, Garches, Grenoble, Lyon avec l'HéliSMUR, le service médical héliporté de la Sécurité civile de Lyon, le Smur Lyon Est, le Smur Lyon Nord, le Smur Lyon Sud, Melun, Montfermeil, Nantes, Orléans, Rennes, Roanne, Roubaix, SaintDenis, Sélestat, Tourcoing et Troyes). Ces Smur couvrent une population à risque d'environ sept millions d'habitants.

Pour les patients atteints par la Covid-19, les cas probables ou confirmés ont été identifiés en accord avec la définition de l'Organisation mondiale de la santé [20]. Cette information a été recueillie ou évaluée par le médecin urgentiste du Smur. Nous avons regroupé les cas probables et confirmés dans le même groupe : Covid.

Le registre RéAC est considéré comme une étude observationnelle prospective. Il a donc obtenu l'approbation du comité consultatif sur le traitement de l'information en matière de recherche dans le domaine de la santé (CCTIRS). Il a également été approuvé par la Commission nationale de l'informatique et des libertés (CNIL — autorisation numéro 910946).

\section{Analyses statistiques}

Nous avons colligé et comparé les caractéristiques épidémiologiques, les délais dont le no-flow (durée de débit cardiaque nul, avant la pratique de tout geste de RCP) et le low-flow (durée de bas débit cardiaque, pendant la pratique de la $\mathrm{RCP}$ ), les gestes de survie (témoins), les gestes de réanimations (SP et Smur) et la survie :

- des patients victimes d'ACEH atteints de la Covid-19 avec les patients non atteints de la Covid-19;

- des ACEH pris en charge suite à un appel au 15 avec ceux ayant appelé le 18 ;

- chez les patients en ACEH atteints de la Covid-19, les patients pris en charge après un appel au 15 avec ceux ayant appelé le 18 .

Les variables quantitatives ont été décrites par les médianes, quartile 1 et quartile 3 . Les variables qualitatives ont été décrites selon les effectifs et les fréquences. Pour les variables de survie, nous avons calculé les intervalles de confiance à $95 \%$ (IC $95 \%$ ). Les comparaisons ont été réalisées en effectuant des tests du $\mathrm{Chi}^{2}$, exacts de Fisher ou U de Mann et Whitney en fonction de la nature des variables. Les analyses statistiques ont été réalisées en situation bilatérale et en utilisant le logiciel SPSS ${ }^{\circledR}$ (version 25.0, IBM $^{\circledR}$, Armonk, NY, États-Unis). Une valeur de $p$ inférieur à 0,05 a été considérée comme significative.

\section{Résultats}

Durant la période d'étude, nous avons inclus 872 patients dont deux pour lesquels l'appel est arrivé par le numéro 112, qui ont été exclus de l'analyse. Ainsi, 870 patients ont été inclus dans l'étude, se répartissant en 184 (21\%) ACEH Covid et 686 (79\%) ACEH non Covid. Concernant la répartition entre appels aux centres 15 et 18 , nous avons obtenu respectivement 487 (56\%) et 383 (44\%) appels (Fig. 1). Les données globales pour ces $870 \mathrm{ACEH}$ mettaient en évidence une RACS de $18 \%$ (IC $95 \%:[15,0 ; 20,5])$, une survie à l'admission de $14 \%$ (IC $95 \%:[11,4 ; 16,3])$ et une survie à j30 de $3 \%$ (IC $95 \%$ : $[2,0 ; 4,2]$ ).

Les patients Covid étaient plus fréquemment de sexe féminin. Les causes d'ACEH étaient plus souvent médicales, et la cause traumatique inexistante (Tableau 1). Le statut Covid du patient ne semblait pas avoir eu d'impact sur le délai d'arrivée des secours (SP ou Smur). En revanche, les délais avant la RACS (ou le décès) du patient ainsi que le délai de no-flow étaient significativement plus longs. Aucun survivant à j30 n'était observé dans le groupe Covid contrairement au groupe non Covid (Tableau 1).

Le tableau 2 compare les caractéristiques, la prise en charge et le devenir des patients victimes d'ACEH suite à un appel au CRRA des Samu ou au CTA des SIS. Lorsque l'appel était destiné au CRRA, les patients étaient plus souvent atteints de la Covid, étaient plus âgés ; les ACEH étaient plus souvent d'étiologie médicale et moins souvent de cause traumatique ou d'une autre cause. Lorsque l'appel était destiné au CRRA, le délai d'arrivée des SP était significativement plus long, et le délai d'arrivée du Smur était plus court. Dans ce groupe, la RCP-B par les SP était moins fréquemment réalisée, et aucune différence significative n'était observée concernant la fréquence de la RCP-S du Smur. Nous n'avons observé aucune différence en termes de survie que l'appel soit passé au 15 ou au 18.

Le tableau 3 montre la même comparaison, 15 vs 18 , pour le sous-groupe des ACEH Covid $(n=115$ [62\%] pour le 15 vs $n=69$ [37\%] pour le 18). Aucune différence significative n'a été observée en termes de caractéristiques des patients, de gestes réalisés par le témoin, de RCP-S par le Smur et de survie. En revanche, dans ce sous-groupe, lorsque l'appel était à destination du 15 , nous avons constaté 


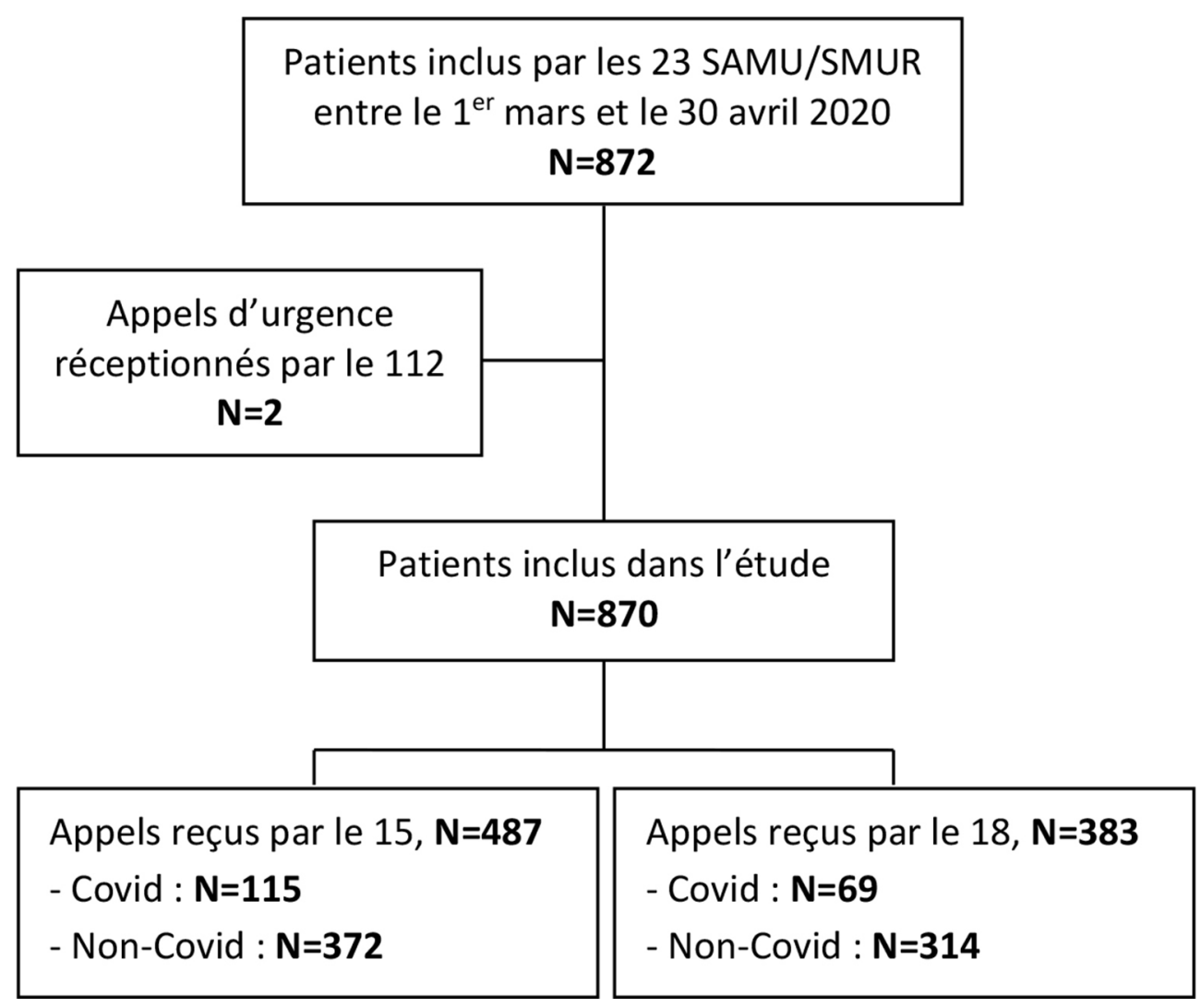

Fig. 1 Diagramme des flux de l'étude

un délai d'arrivée des SP plus long et un taux de RCP-B par les SP plus faible.

\section{Discussion}

Notre étude a permis de décrire et d'analyser les particularités des victimes d'ACEH et de leur prise en charge lors de l'épisode de suractivité lié à la pandémie de Covid-19 dont l'acmé s'est située pour l'ensemble des Samu français entre début mars et fin avril 2020. Ainsi, cette étude n'avait pas pour vocation première de comparer les caractéristiques des $\mathrm{ACEH}$ et de leur traitement par rapport aux années antérieures, hors période épidémique, comme d'autres études ont pu le réaliser [21]. Plusieurs résultats ont été mis en lumière.

Le premier résultat concerne le devenir des ACEH au cours de cette période épidémique dont les principaux paramètres de survie paraissent inférieurs aux données les plus récentes issues du registre RéAC : la RACS était de $18 \%$, la survie à l'admission de $14 \%$ et à j30 de $3 \%$, alors qu'une vaste étude sur une population de $18185 \mathrm{ACEH}$ inclus dans le registre RéAC entre 2012 et 2018 observait $32 \%$ de RACS, avec une survie à l'admission de $27 \%$ et à j30 de $7 \%$ [22]. Cette diminution d'environ la moitié de la survie de l'ensemble des ACEH sur l'ensemble du territoire fran- çais, quelle qu'en soit l'origine, confirme la tendance globale préalablement rapportée de façon limitée à Paris et sa petite couronne [21].

Lorsque nous comparons les ACEH survenant chez des patients porteurs et/ou suspects d'être Covid avec les patients non atteints, nous observons plus d'ACEH d'origine médicale (avec une disparition des ACEH traumatiques dans ce groupe Covid), ce qui peut s'expliquer par l'atteinte virale sous-jacente ainsi que par une tendance vers une surreprésentation des ACEH survenus au domicile en lien probable avec la quatorzaine imposée à ces patients contagieux.

Dans le groupe Covid, la durée du no-flow, c'est-à-dire la période avant que ne débute un premier massage cardiaque, est plus importante, reflétant indiscutablement un retard dans la mise en place des premiers gestes de survie par les témoins dans ce contexte de contagion. Cependant, la fréquence des gestes réalisés par le témoin n'est pas différente entre les patients Covid et non Covid. Cela est encore une fois le reflet de la quarantaine et de la survenue des ACEH au domicile, le témoin étant, sans doute, plus souvent un membre de la famille : ainsi, il s'agit probablement de témoins familiaux non formés dont les délais pour la mise en place des gestes de survie sont longs indépendamment des risques de contagion qui passent alors au second plan et n'empêchent pas finalement la réalisation des premiers gestes de survie, 
Tableau 1 Comparaison des arrêts cardiaques extrahospitaliers (ACEH) chez les patients Covid et non Covid entre le $1^{\text {er }}$ mars et le 30 avril 2020 dans le registre RéAC

\begin{tabular}{|c|c|c|c|}
\hline Variables & $\begin{array}{l}\text { Covid } \\
(n=184)\end{array}$ & $\begin{array}{l}\text { Non-Covid } \\
(n=686)\end{array}$ & Valeur de $p$ \\
\hline Âge (années) & $70[58 ; 83]$ & $70[56 ; 82]$ & 0,90 \\
\hline Sexe & & & 0,03 \\
\hline Masculin & $114(62 \%)$ & $484(71 \%)$ & \\
\hline Féminin & $70(48 \%)$ & $202(39 \%)$ & \\
\hline Survenue à domicile & $160(87 \%)$ & $560(82 \%)$ & 0,01 \\
\hline Cause de l'ACEH & & & $<0,01$ \\
\hline Médicale & $181(98 \%)$ & $574(84 \%)$ & \\
\hline Traumatique & $0(0 \%)$ & $41(6 \%)$ & \\
\hline Autres & $3(2 \%)$ & $71(10 \%)$ & \\
\hline Gestes de survie initiés par les témoins & $86(47 \%)$ & $318(46 \%)$ & 0,93 \\
\hline Délai arrivée premiers secours (min) & $12[6 ; 17]$ & $10[7 ; 14]$ & 0,11 \\
\hline Délai arrivée Smur (min) & $20[14 ; 31]$ & $19[14 ; 27]$ & 0,27 \\
\hline Délai avant RACS (min) & $42[28 ; 58]$ & $37[25 ; 50]$ & 0,03 \\
\hline No-flow $(\mathrm{min})$ & $14[6 ; 22]$ & $11[5 ; 19]$ & 0,05 \\
\hline Low-flow (min) & $26[10 ; 44]$ & $24[11 ; 38]$ & 0,31 \\
\hline Réanimation de base initiée par les SP & $151(82 \%)$ & $522(76 \%)$ & 0,09 \\
\hline Réanimation médicale initiée par le Smur & $99(54 \%)$ & $395(58 \%)$ & 0,36 \\
\hline RACS & $28(15 \%)$ & $125(18 \%)$ & 0,38 \\
\hline Survie à l'admission & $18(10 \%)$ & $101(15 \%)$ & 0,09 \\
\hline Survie à J30 & $0(0 \%)$ & $24(4 \%)$ & $<0,01$ \\
\hline Valeurs manquantes & 5 & 29 & \\
\hline État neurologique favorable à $\mathrm{J} 30$ & NA & $19(95 \%)$ & NA \\
\hline Valeurs manquantes & & 4 & \\
\hline
\end{tabular}

souvent conseillés par téléphone. Le dernier paramètre significatif est la durée de réanimation pour obtenir une éventuelle RACS qui est plus longue dans le groupe Covid en lien probable avec une réanimation plus compliquée due aux difficultés de prise en charge et/ou à la gravité de la pathologie virale elle-même. Le reste des paramètres épidémiologiques (délais d'arrivée des prompts secours, des Smur, nombre de réanimations médicales initiées et durée de réanimation) ne montre pas de différence significative. Enfin, si aucun critère de pronostic n'est significativement différent, il existe une tendance en défaveur des ACEH Covid par comparaison avec les ACEH non Covid, notamment en termes de survie à l'admission ( 15 vs $10 \%, p=0,09)$, et l'absence de survivant à j30 lorsque le patient est Covid.

Par ailleurs, nous avons observé que près de $56 \%$ des appels pour ACEH étaient à destination du numéro 15 et près de $44 \%$ pour le 18 . Sur la période d'étude, seuls deux appels étaient à destination du 112, démontrant la méconnaissance quasi totale en France de ce numéro d'appel en cas d'urgence vitale. Cette répartition des appels plus importante au centre 15 était majorée lorsque l'ACEH concernait un patient Covid. Au-delà de cette constatation, lorsqu'un appel était à destination du centre 15 , en comparaison avec le 18, essentiellement trois paramètres apparaissaient significativement différents : il y avait plus d'ACEH d'origine médicale et de patients Covid, alors qu'il y avait plus d'ACEH d'origine traumatique dans les appels au 18. L'arrivée des prompts secours était significativement plus rapide, et le no-flow était significativement inférieur lors des appels au 18. Cette différence peut s'expliquer par un déclenchement plus rapide des prompts secours du fait d'absence ou d'insuffisance de moyens de communication prioritaire et d'interconnexions entre les CTA et les CRRA dans certains secteurs du territoire, ce qui devrait être corrigé par le déploiement national prochain des nouveaux systèmes d'information interconnectés des Samu et des SIS, et du service d'accès aux soins (SAS) qui devrait, notamment, offrir une harmonisation des outils et des interfaces CTA/CRRA. 
Tableau 2 Comparaison des arrêts cardiaques extrahospitaliers (ACEH) selon le numéro d'appel (15 vs 18$)$ entre le $1^{\text {er }}$ mars et le 30 avril 2020 dans le registre RéAC

\begin{tabular}{|c|c|c|c|}
\hline Variables & $\begin{array}{l}\text { Appel 15 } \\
(n=487)\end{array}$ & $\begin{array}{l}\text { Appel } 18 \\
(n=383)\end{array}$ & Valeur de $p$ \\
\hline Patients Covid & $115(24 \%)$ & $69(18 \%)$ & 0,04 \\
\hline Âge (années) & $71[59 ; 83]$ & $68[55 ; 81]$ & $<0,01$ \\
\hline Sexe & & & 0,06 \\
\hline Masculin & $321(66 \%)$ & $277(72 \%)$ & \\
\hline Féminin & $166(34 \%)$ & $106(28 \%)$ & \\
\hline Survenue à domicile & $406(83 \%)$ & $314(82 \%)$ & 0,59 \\
\hline Cause de l'ACEH : & & & $<0,01$ \\
\hline Médicale & $441(90 \%)$ & $314(82 \%)$ & \\
\hline Traumatique & $12(2 \%)$ & $29(8 \%)$ & \\
\hline Autres & $34(7 \%)$ & $40(10 \%)$ & \\
\hline Gestes de survie initiés par les témoins & $225(46 \%)$ & $179(47 \%)$ & 0,89 \\
\hline Délai arrivée premiers secours (min) & $13[7 ; 17]$ & $9[6 ; 12]$ & $<0,01$ \\
\hline Délai arrivée Smur (min) & $18[13 ; 28]$ & $20[15 ; 28]$ & 0,01 \\
\hline Délai avant RACS (min) & $38[25 ; 54]$ & $37[27 ; 50]$ & 0,37 \\
\hline No-flow (min) & $14[5 ; 20]$ & $11[4 ; 19]$ & 0,02 \\
\hline Low-flow (min) & $24[10 ; 39]$ & $25[12 ; 40]$ & 0,66 \\
\hline Réanimation de base initiée par les SP & $356(73 \%)$ & $317(83 \%)$ & $<0,01$ \\
\hline Réanimation médicale initiée par le Smur & $279(57 \%)$ & $215(56 \%)$ & 0,78 \\
\hline RACS & $88(18 \%)$ & $65(17 \%)$ & 0,72 \\
\hline Survie à l'admission & $66(14 \%)$ & $53(14 \%)$ & 0,92 \\
\hline Survie à J30 & $14(3 \%)$ & $10(3 \%)$ & 0,99 \\
\hline Valeurs manquantes & 16 & 18 & \\
\hline État neurologique favorable à $\mathrm{J} 30$ & $11(92 \%)$ & $8(100 \%)$ & 0,99 \\
\hline Valeurs manquantes & 2 & 2 & \\
\hline
\end{tabular}

On constate également que lorsque les appels arrivent au CTA (18), la RCP-B était plus souvent initiée par les équipes des SP que lorsque l'appel était régulé par le CRRA (15), alors qu'on ne retrouve pas de différence significative dans l'initiation et la réalisation de la réanimation médicale (RCP$\mathrm{S})$, ce qui pourrait être en lien avec une arrivée plus précoce du Smur ne laissant pas le temps à la prise en charge secouriste. La survie, quant à elle, est comparable à tous les stades d'analyse du pronostic pour l'ensemble des ACEH survenus dans cette période de suractivité des Samu/Smur, quel que soit le numéro d'appel choisi. Ce résultat a été confirmé pour le sous-groupe des ACEH Covid pour lesquels le pronostic ne dépendait pas, non plus, du numéro appelé.

Notre étude comporte quelques limites. La première est liée au registre RéAC. Ce registre est fondé sur la participation volontaire des Samu/Smur français. La participation des Smur au RéAC n'est pas obligatoire. Les Smur qui choisissent de participer au RéAC n'incluent pas de manière systématique tous leurs cas d'ACEH. Ainsi, une sélection des Smur a été obligatoire pour obtenir une exhaustivité des inclusions la plus proche possible de $100 \%$ assurant des calculs de haute fiabilité. Certes, cette étude ne permet pas de couvrir la population française entière. Cependant, les Smur inclus couvrent des populations représentatives du territoire (milieu rural et urbain). De plus, les Smur sélectionnés couvrent une population à risque d'environ sept millions d'habitants, soit plus de $10 \%$ de la population française. La seconde limite relève de la problématique du statut virologique du patient. En effet, certains patients ont pu être classés dans la mauvaise catégorie (Covid ou non Covid), car ne disposant pas des informations post-mortem certains patients non Covid ont pu être des faux-négatifs. De plus, les limites d'accès aux tests de Covid-19 en France, principalement en début de pandémie, ont sans doute entraîné une sous-évaluation du nombre de cas Covid-19. 
Tableau 3 Comparaison des arrêts cardiaques extrahospitaliers (ACEH) chez les seuls patients Covid entre le $1^{\mathrm{er}}$ mars et le 30 avril 2020 dans le registre RéAC

\begin{tabular}{|c|c|c|c|}
\hline Variables & $\begin{array}{l}\text { Appel } 15 \\
(n=115)\end{array}$ & $\begin{array}{l}\text { Appel } 18 \\
(n=69)\end{array}$ & Valeur de $p$ \\
\hline Âge (années) & $70[58 ; 82]$ & $70[58 ; 83]$ & 0,82 \\
\hline Sexe & & & 0,12 \\
\hline Masculin & $66(57 \%)$ & $48(70 \%)$ & \\
\hline Féminin & $49(43 \%)$ & $21(30 \%)$ & \\
\hline Survenue à domicile & $97(84 \%)$ & $63(91 \%)$ & 0,26 \\
\hline Cause de l'ACEH & & & 0,99 \\
\hline Médicale & $113(98 \%)$ & $68(99 \%)$ & \\
\hline Traumatique & $0(0 \%)$ & $0(0 \%)$ & \\
\hline Autres & $2(2 \%)$ & $1(1 \%)$ & \\
\hline Gestes de survie initiés par les témoins & $52(45 \%)$ & $34(49 \%)$ & 0,65 \\
\hline Délai arrivée premiers secours (min) & $15[8 ; 20]$ & $10[5 ; 15]$ & 0,02 \\
\hline Délai arrivée Smur (min) & $20[13 ; 33]$ & $20[15 ; 27]$ & 0,86 \\
\hline Délai avant RACS (min) & $43[27 ; 60]$ & $40[29 ; 55]$ & 0,70 \\
\hline No-flow (min) & $15[8 ; 24]$ & $12[5 ; 18]$ & 0,04 \\
\hline Low-flow (min) & $27[10 ; 42]$ & $25[12 ; 49]$ & 0,58 \\
\hline Réanimation de base initiée par les SP & $89(77 \%)$ & $62(90 \%)$ & 0,04 \\
\hline Réanimation médicale initiée par le Smur & $64(56 \%)$ & $35(51 \%)$ & 0,54 \\
\hline RACS & $19(16 \%)$ & $9(13 \%)$ & 0,67 \\
\hline Survie à l'admission & $11(10 \%)$ & $7(10 \%)$ & 0,99 \\
\hline Survie à $\mathrm{J} 30$ & $0(0 \%)$ & $0(0 \%)$ & NA \\
\hline Valeurs manquantes & 3 & 2 & \\
\hline
\end{tabular}

\section{Conclusion}

Cette étude des ACEH à partir des données du Registre (RéAC) permet une analyse objective de la prise en charge des ACEH pendant la crise sanitaire sans précédent et confirme que le pronostic des ACEH sur cette période s'est fortement dégradé par rapport aux données antérieures issues du même registre. Cette étude montre une réduction supplémentaire de survie dans le groupe Covid, avec notamment aucun survivant à $\mathrm{j} 30$. A contrario, le pronostic de tous les ACEH de cette période, comme le pronostic des seuls ACEH Covid, est équivalent quel que soit le service appelé (15 ou 18), le délai d'arrivée et de mise en action des prompts secours, noté plus court en cas d'appel initial au 18, n'ayant pas eu d'impact sur les chances de survie.

Liens d'intérêts : les auteurs déclarent ne pas avoir de liens d'intérêts.

\section{Références}

1. Riou B (2020) Retour d'expérience de la crise Covid-19 en médecine d'urgence. Ann Fr Med Urg 10:197-8

2. Garcia-Castrillo L, Petrino R, Leach R, et al (2020) European Society For Emergency Medicine position paper on emergency medical systems' response to Covid-19. Eur J Emerg Med 27:174-7

3. Rothan HA, Byrareddy SN (2020) The epidemiology and pathogenesis of coronavirus disease (Covid-19) outbreak. J Autoimmun 109: 102433

4. Bai Y, Yao L, Wei T, et al (2020) Presumed asymptomatic carrier transmission of COVID-19. JAMA 323:1406-7

5. Mejicano GC (1998) Infections acquired during cardiopulmonary resuscitation: Estimating the risk and defining strategies for prevention. Ann Intern Med 129:813

6. Christian MD, Loutfy M, McDonald LC, et al (2004) Possible SARS coronavirus transmission during cardiopulmonary resuscitation. Emerg Infect Dis 10:287

7. Liu W, Tang F, Fang LQ, et al (2009) Risk factors for SARS infection among hospital healthcare workers in Beijing: a case control study. Trop Med Int Health 14:52-9 
8. Berg RA, Hemphill R, Abella BS, et al (2010) Part 5: adult basic life support: 2010 American Heart Association guidelines for cardiopulmonary resuscitation and emergency cardiovascular care. Circulation 122:S685-S705

9. Perkins GD, Handley AJ, Koster RW, et al (2015) European Resuscitation Council guidelines for resuscitation 2015: Section 2. Adult basic life support and automated external defibrillation. Resuscitation 95:81-99

10. Couper K, Taylor-Phillips S, Grove A, et al (2020) Covid-19 in cardiac arrest and infection risk to rescuers: a systematic review. Resuscitation 151:59-66

11. Soar J, Nolan JP, Böttiger BW, et al (2015) Adult advanced life support section collaborators. European Resuscitation Council guidelines for resuscitation 2015: Section 3. Adult advanced life support. Resuscitation 95:100-47

12. Perkins GD, Morley PT, Nolan JP, et al (2020) International Liaison Committee on Resuscitation: COVID-19 consensus on science, treatment recommendations and task force insights. Resuscitation 151:145-7

13. Groupe de travail de Samu Urgences de France (SUdF) et du Conseil français de réanimation cardiopulmonaire (CFRC) (2020) Recommandations concernant la protection des personnels et la prise en charge préhospitalière des arrêts cardiorespiratoires de patients adultes suspects ou confirmés d'infections à Covid19. https://www.samu-urgences-de-france.fr/medias/files/Recommandations\%20SUdFCFRC_ACR\%20et\%20Covid_200425.pdf (Dernier accès le 20 octobre 2020)

14. Mahase E, Kmietowicz Z (2020) Covid-19: doctors are told not to perform CPR on patients in cardiac arrest. BMJ 368:m1282

15. Adnet F, Lapostolle F (2004) International EMS Systems: France. Resuscitation 63:7-9
16. Hubert H, Tazarourte K, Wiel E, et al (2014) Rationale, methodology, implementation, and first results of the French out-of-hospital cardiac arrest registry, Prehospit Emerg Care 18:511-9

17. Perkins GD, Jacobs IG, Nadkarni VM, et al (2015) Cardiac arrest and cardiopulmonary resuscitation outcome reports: update of the Utstein resuscitation registry templates for out-of-hospital cardiac arrest: a statement for healthcare professionals from a task force of the International Liaison Committee on Resuscitation (American Heart Association, European Resuscitation Council, Australian and New Zealand Council on Resuscitation, Heart and Stroke Foundation of Canada, InterAmerican Heart Foundation, Resuscitation Council of Southern Africa, Resuscitation Council of Asia); and the American Heart Association Emergency Cardiovascular Care Committee and the Council on Cardiopulmonary, Critical Care, Perioperative and Resuscitation. Circulation 132:1286-300

18. Gueugniaud PY, Bertrand C, Savary D, Hubert H (2011) L'arrêt cardiaque en France : pourquoi un registre ? Presse Med 40:634-8

19. The Brain Resuscitation Clinical Trial I Study Group (1986) Randomized clinical study of thiopental loading in comatose survivors of cardiac arrest. N Engl J Med 314:397-403

20. World Health Organization (2020) Global surveillance for Covid-19 caused by human infection with Covid-19 virus: interim guidance. https://apps.who.int/iris/handle/10665/331506 (Dernier accès le 17 octobre 2020)

21. Marijon E, Karam N, Jost D, et al (2020) Out-of-hospital cardiac arrest during the Covid-19 pandemic in Paris, France: a populationbased, observational study. Lancet Public Health 5:e437-e443

22. Noel L, Jaeger D, Baert V, et al (2019) Effect of bystander CPR initiated by a dispatch centre following out-of-hospital cardiac arrest on 30-day survival: Adjusted results from the French national cardiac arrest registry. Resuscitation 144:91-8 